\title{
Le théâtre de Césaire, écritures-réécritures, un travail de Sisyphe
}

Daniel Delas

\section{OpenEdition Journals}

Édition électronique

URL : http://journals.openedition.org/coma/692

DOI : $10.4000 /$ coma.692

ISSN : 2275-1742

\section{Éditeur}

Institut des textes \& manuscrits modernes (ITEM)

\section{Référence électronique}

Daniel Delas, "Le théâtre de Césaire, écritures-réécritures, un travail de Sisyphe », Continents manuscrits [En ligne], 7| 2016, mis en ligne le 15 décembre 2016, consulté le 19 avril 2019. URL http://journals.openedition.org/coma/692 ; DOI : 10.4000/coma.692

Ce document a été généré automatiquement le 19 avril 2019

\section{(i) $\odot$

Continents manuscrits - Génétique des textes littéraires - Afrique, Caraîbe, dispora est mis à disposition selon les termes de la licence Creative Commons Attribution - Pas d'Utilisation

Commerciale - Pas de Modification 4.0 International. 


\title{
Le théâtre de Césaire, écritures- réécritures, un travail de Sisyphe
}

\author{
Daniel Delas
}

1 Les travaux sur le théâtre de Césaire sont nombreux et ont fourni des bases de réflexion solides pour en comprendre les tenants et les aboutissants, les enjeux et les risques, les problématiques littéraires, culturelles et politiques. Ces études se sont souvent appuyées sur des propos d'Aimé Césaire lui-même, très généreux de son temps, tout au long de sa vie, avec les jeunes chercheurs et très ouvert aux entretiens avec des journalistes. Beaucoup des acteurs qui ont joué son théâtre, la plupart des metteurs en scène de ses pièces ont eux aussi écrit de précieux souvenirs ou donné de fécondes interviews. Rassembler tous ces documents en une sorte d' "hyper-Césaire dramaturge » permettrait assurément d'avoir une meilleure vue d'ensemble d'une œuvre complexe et en constant renouvellement. En prenant garde toutefois - et ce n'est pas la moindre des difficultés de cette entreprise - de ne pas cloisonner poésie / théâtre / essais et discours. Car pour ne prendre qu'un exemple, le Discours sur le colonialisme contient dans son premier élargissement de 1950, une satire bouffonne de certaines séances de la Chambre des Députés très proche des textes de théâtre qu'il écrit à cette époque pour la version théâtrale de Et les chiens se taisaient ou pour les premières feuilles de La tragédie du roi Christophe. ${ }^{1}$ Tous les textes politiques de Césaire se tiennent étroitement et la barrière des genres est dans leur cas très poreuse.

2 Le récent recensement des écrits de Césaire fait par K. Veron et Th. Hale réunit de nombreux éléments, sinon nouveaux, toutefois auparavant dispersés ${ }^{2}$. Diverses entreprises éditoriales ont apporté beaucoup de matériau génétique, en particulier le volume qu'a dirigé James Arnold ${ }^{3}$.

3 Mon propos n'est pas de reprendre toute cette matière pour en produire une sorte de digest critique mais plutôt de m'appuyer sur certains de ses apports pour tenter de répondre à la question: Césaire n'a-t-il tant écrit et tant réécrit ses textes de théâtre que parce que, au delà des exempla offerts par l'histoire, un seul sujet lui tenait à cœur, celui de la libération de l'homme ? L'homme noir certes puisqu'il est historiquement le premier opprimé mais, potentiellement, l'homme, l'homme tout court. En somme n'a-t-il jamais 
écrit que le même texte? Repris, remanié, adapté, remodelé selon les publics et les fonctions? En quête d'un théâtre total où pourraient s'intégrer les spécificités culturelles des peuples ? Rêverie utopique ou combat concret d'une vie?

4 Parlons-en.

Certains écrivains se sont rendus célèbres par un seul livre et s'en sont tenus là ou n'ont plus jamais retrouvé l'inspiration «miraculeuse » de leur premier écrit : pour nous en tenir à des écrivains afro-antillais, citons: Cheikh Hamidou Kane (L'aventure ambiguë), Yambo Ouologem (Le devoir de violence), Sonny Rupaire (Cette igname brisée qu'est ma terre natale). D'autres, bien plus nombreux heureusement, ayant commencé à écrire, continueront de le faire, malgré ou à cause de l'épreuve qu'est l'écriture. Pourquoi ? On pourra répondre en distinguant deux catégories a priori distinctes d'écrivains. D'un côté, ceux qui écrivent aiguillonnés par une curiosité historique et anthropologique insatiable (Lafcadio Hearn ou Albert Londres hier, Jean-Christophe Rufin, Pierre Assouline ou JeanPaul Kauffmann aujourd'hui), qui sont, au sens le meilleur, des écrivains journalistes ${ }^{4}$ - et qui en vivent! Les écrivains dits du voyage (Nicolas Bouvier ou Colin Thubron) exemplifient une autre branche de cette " horde du vécu ", très puissante aujourd'hui en raison de ce "goût de l'autre » qui incite les amateurs à acheter leurs livres. Je les lis d'ailleurs pour ma part avec gourmandise, avec le sentiment de m'enrichir d'un regard écologique nouveau sur des mondes en voie de disparition. D'un autre côté des écrivains poètes qui pensent nécessaire d'inventer des langages neufs pour dire un monde nouveau : écrivains engagés, écrivains révoltés, écrivains rêveurs et combatifs. Arrêtonsnous sur le théâtre de Césaire qui est un remarquable représentant de cette dernière catégorie. Certes, disait-il d'ailleurs, il m'est arrivé d'écrire des pièces de théâtre, des drames, des tragédies, mais pour moi ce sont des « départements » de la poésie :

En effet, qu'est-ce qu'un poète? Selon la définition de Rimbaud, c'est un voyant ; par conséquent, le poète qui a pour qualité première de faire voir, de voir pour son propre compte, devient homme de théâtre dès le moment où il essaie de faire voir, de transmettre sa vision aux autres. ${ }^{5}$

5 Dans la présentation de la section théâtre du volume à dominante critique et génétique cité plus haut, James Arnold écrit à propos de ECT:

Il y a une vingtaine d'années on pouvait croire que la vocation théâtrale d'Aimé Césaire remontait à 1955 et au projet d'adaptation par Janheinz Jahn de la tragédie Et les chiens se taisaient pour la scène germanique. Depuis la découverte en 2008 d'un drame historique, nous savons que la révolution de Saint Domingue préoccupait Césaire depuis $1941^{6}$.

6 Autrement dit, à l'instar de l'écriture du CRPN qui s'étale sur plus de quinze ans - éditions de 1939 (Volontés), Brentano's (1943), Bordas (1947), Présence africaine (1956) -, l'élaboration de $E C T$ couvre, quant à elle, plus de dix années depuis le premier «drame nègre » de 1943 jusqu'à la tragédie de 1956, stabilisée avec l'aide de Janheinz Jahn, en passant par l'oratorio lyrique joint au recueil de poèmes Les Armes miraculeuses en 1946. Puis l'entreprise se poursuit avec les écritures et réécritures qui aboutiront à la version finale de TRC en 1970. Pendant près de trente ans donc, Césaire est revenu sans trêve sur la question du leader noir des indépendances afro-antillaises depuis Toussaint Louverture et Christophe jusqu'à Patrice Lumumba et Mobutu. 
7 Du tapuscrit du premier état de ECT au drame lyrique de 1946, on ne constate pas moins de soixante-quatre transpositions ${ }^{7}$ qui se sont faites en plusieurs étapes, en fonction de la réflexion de Césaire sur l'évolution de la situation politique; laquelle s'infléchit, de Toussaint à Christophe, d'une représentation historienne ${ }^{8}$ du rôle et du personnage de Toussaint-Louverture, dont on se rappelle qu'il est présent dans le Cahier ${ }^{9}$ :

c'est un homme seul emprisonné de blanc

c'est un homme seul qui défie les cris blancs de la mort blanche

(TOUSSAINT, TOUSSAINT LOUVERTURE)

c'est un homme seul qui fascine l'épervier blanc de la mort blanche

c'est un homme seul dans la mer inféconde de sable blanc ${ }^{10}$

à une représentation marquée d'historicité, qui veut faire de Toussaint et de Christophe, au delà des personnages historiques qu'ils furent, à la fois l'exemple grandiose de celui qui se sacrifie hier, aujourd'hui et demain pour l'humanité à venir et qui pourtant, en un contrepoint ombreux, incarne peut-être aussi l'homme que stigmatise le jugement des peuples noirs comme un mégalomane en marche vers la dictature. Il y a une importante évolution interne entre la version lyrique de ECT dans Les armes miraculeuses et la version historique qui se concrétisera théâtralement avec TRC dont deux états sont édités, en 1963 et en 1970.

8 Cette histoire permet de mieux comprendre comment s'affrontent sans fin, dans la genèse de l'ensemble du projet théâtral césairien, deux pratiques d'écriture dont la combinatoire est problématique, l'une lyrique et poétique, l'autre dramatique et satirique. Césaire l'a d'ailleurs reconnu lui-même ${ }^{11}$ en disant qu'il avait voulu " concilier Nietzsche et Brecht, Shakespeare et les rituels africains ». Tâche difficile puisqu'à ne considérer que les deux premiers, leurs propositions sont contradictoires, Nietzsche mettant l'emphase sur la violence brûlante et salutaire de la force dionysiaque, d'origine barbare (orientale) pour les Grecs, Brecht, au contraire soumettant la représentation de cette force à l'exigence " refroidissante » d'une distanciation qui permette d'en avoir une vision claire. La version 1946 de ECT est écrite dans le sillage d'une relecture des tragiques grecs à la lumière de Nietzsche, mettant en scène « la Grèce primitive, barbare et rude, pas la Grèce alexandrine ", forme d'art "très près de l'Afrique ", d'où les allusions aux sacrifices rituels, inspirés des mythes égyptiens et aux rites d'Osiris qui disparaissent dans la version de 1956 et n'apparaissent bien entendu nullement dans la TRC.

9 La réécriture de ECT en 1956 apparaît ainsi comme une étape intermédiaire vers la théâtralisation pédagogique de l'histoire de Toussaint-Louverture/Christophe que Césaire entreprend d'écrire dès le début des années 60. Car la leçon de Nietzsche dans la Naissance de la tragédie ne se réduit pas à la dichotomie féconde dionysiaque / apollinien, il faut aussi rappeler que selon le philosophe allemand, la naissance d'un peuple doit s'accompagner de la naissance d'un théâtre : «la tragédie antique comme institutrice du peuple », écrit-i ${ }^{12}$. Bien avant sa démission du Parti communiste en 1956, Césaire tournait et retournait dans sa tête une interrogation importante : au delà de la poésie qui est sa respiration même, au delà du discours politique qui est pris dans l'urgence et dans le compromis (même si les textes politiques sont essentiels pour le combat), le théâtre n'estil pas le meilleur moyen de toucher le peuple, qui ne lit ni la poésie ni les romans et n'écoute que d'une oreille les discours politiques?

10 Ce qui conduit à une réflexion, qui semble secondaire mais qui ne l'est pas, sur l'impossibilité de stabiliser définitivement les textes théâtraux de Césaire.

L'édition de 1970, commente James Arnold, représente, non une version définitive, mais plutôt l'état qui résulta de six ans de travail intense de collaboration 
collective, associant des comédiens tels que Douta Seck aux modifications proposées soit par Serreau soit par Césaire. Il s'agit donc d'un document théâtral repris par l'auteur et qui deviendra œuvre littéraire à la suite de son édition chez Présence Africaine. ${ }^{13}$

Le primat de l'écrit en Occident (son grammatocentrisme) a été illustré et sans doute amplifié par l'école telle qu'elle s'est développée en France. Pour enseigner la langue, on en a inventé la grammaire; pour enseigner la littérature, on a constitué un patrimoine écrit, chaque texte étant un monument intangible dont on extrayait des fragments appelés à constituer la mémoire nationale. Quant au théatre, il s'enseignait à l'école à partir de textes écrits. Cette solidification de la langue et de la littérature a été bien étudiée à partir des années 70 (travaux de Macherey, de Balibar, de Chervel et bien d'autres) et on le rappelle ici pour bien souligner que Césaire inscrit son travail totalement à rebours d'une tradition française bien installée.

11 Césaire, on l'a vu, reprend, remanie, réécrit sans cesse. Ce que l'on voudrait ajouter, c'est qu'il poursuit dans le même sens en participant au travail de mise en scène de ses pièces. Ce qui ne va pas sans lui causer quelque inquiétude. On connaît le petit dialogue révélateur entre le metteur en scène Jean-Marie Serreau et l'auteur Aimé Césaire, le premier disant « " Le texte de théâtre n'est pas du bronze », le second répondant, un peu inquiet tout de même, « mais il ne faut pas que ce soit du sable $»^{14}$.

12 La résistance de l'auteur / écrivain Césaire n'empêche pas que la TRC ait connu deux versions (1963 et 1970) et que les modifications introduites dans la deuxième version n'aient pas été faites dans un « tête-à-texte » (P.Kuentz) retiré du bruit du monde mais en présence / au milieu de ce bruissement de paroles politiques qui disent la vie des hommes en société, et plus particulièrement dans le rythme même de la mise en scène et du jeu des acteurs. Serreau étant un metteur en scène engagé, profondément brechtien, et sa troupe étant composée d'Africains au premier rang desquels brillait Douta Seck, les modifications qu'accepte Césaire viennent autant d'une réflexion proprement politique que d'une négrification consentie de son texte, nourrie en particulier des apports des acteurs noirs de la troupe. Comme à son habitude, Césaire procède de diverses manières (suppressions / adjonctions / permutations) qui ajustent la portée de l'analyse critique et politique d'une part et accentuent d'autre part le caractère africain par une insistance accrue sur ce qui provient chez Christophe de la culture haïtienne, où le vaudou joue un rôle important.

Toutes les remarques que nous venons de faire sur TRC peuvent s'appliquer mutatis mutandis à Une saison au Congo, texte qui a connu lui aussi une genèse interactive et poursuit la quête critique du dramaturge martiniquais sur le rôle du leader révolutionnaire noir, poteau mitan du théâtre césairien.

13 Le texte de théâtre césairien est progressivement devenu un palimpseste, on l'a compris, toujours plus «poreux à tous les souffles du monde " (pour reprendre les termes du Cahier), à mesure que l'histoire du temps présent afro-antillais l'infiltrait et le transformait. Si l'on met à part Une tempête plus éloignée des réalités historiques, les trois autres pièces de Césaire sont très étroitement connectées à l'histoire contemporaine des indépendances africaines et à la culture noire. Résultant de la réflexion en mouvement de l'auteur, elles sont aussi associées et modifiées par la recherche de nouvelles formes dramatiques que l'expression « théâtre total » résume assez bien.

Ce que je voudrais - dit-il en 1966, à l'occasion du Festival mondial des arts nègres -, c'est créer un théâtre noir (... ). Ce doit être un théâtre total. Il doit réunir tous les 
arts et en être la synthèse et la symbiose. Il doit être différent de la forme de théâtre que l'Europe connaît en ce moment. ${ }^{15}$

14 Césaire est en phase avec des metteurs en scène novateurs de son temps, comme JeanLouis Barrault, qui utilisent beaucoup cette expression. On peut aussi citer Maurice Béjart, qui déclare en 1965 :

Je crois que nous nous dirigeons vers un théâtre collectif, je crois que l'époque du créateur isolé, de l'artiste qui s'enferme dans sa chambre pendant six mois, un an ou deux, est révolue. Je ne dis pas que cette attitude n'est pas valable, mais elle ne correspond plus à notre mentalité actuelle et le théâtre total sera une œuvre collective. ${ }^{16}$

15 On comprend désormais mieux pourquoi la TRC est la seule œuvre "étrangère » du répertoire du Rocado Zulu Théâtre de Sony Labou Tansi ${ }^{17}$, lequel, à l'instar de Molière, a écrit toutes les autres pièces du répertoire de la troupe, sauf précisément celle-ci. Césaire, en acteur politique qu'il était d'abord, espérait agir par ses trois pièces, en dénonçant les mauvaises forces (blanches autant que noires) acharnées à étouffer tout mouvement révolutionnaire. Il voulait aussi «conscientiser» (mot nkrumien alors en vogue) les peuples contre la mégalomanie et la paranoïa des dictateurs idéologues comme Mobutu ou Sékou Touré. Comme Brecht avait tenté de le faire à propos de Hitler. Le théâtre de Césaire est donc militant alors que celui de Sony s'écrit dans la continuité de mais en réaction à celui de Césaire, dans une africanité plus radicale mais éloignée de tout militantisme. Sony pense que le théâtre de Césaire a lancé le mouvement théâtral africain dans la bonne direction, critique et burlesque, mais qu'il reste encore trop bourgeois, «à l'occidentale ", confiné en salle ou sur scène. En une période de reflux de toutes les certitudes occidentales, il convient selon lui de faire exploser les rituels qui brident la force de l'expression tant sur le plan du langage que sur celui de l'espace ${ }^{18}$. Parce que la tâche essentielle de l'écrivain est d'abord d'aider à voir autrement l'homme et le monde. Non plus donc un théâtre qui milite en faveur d'un homme idéal qui n'existe pas et n'existera jamais, mais un théâtre qui agit philosophiquement au niveau de l'homme tout entier.

Comme le dit Dieudonné Niangouna :

Le temps des spectacles est passé. Même la nudité ne dit plus la vérité. Nous allons fermer le théâtre pour ouvrir l'homme du dedans. ${ }^{19}$

\section{NOTES}

1. On utilisera par la suite les abréviations suivantes: CRPN pour le Cahier d'un retour au pays natal, ECT pour Et les chiens se taisaient, TRC pour La tragédie du roi Christophe.

2. Les Ecrits d'Aimé Césaire, biobibliographie commentée (1913-2008), 2 volumes, Paris, Honoré Champion éditeur, 2013, 891 pages. 
3. Aimé Césaire, Poésie, Théâtre, Essais et Discours, CNRS éditions / Présence africaine éditions, «Planète libre » édition critique, coordinateur Albert James Arnold, 2013, 1805 pages.

4. Gabriel Garcia Marquez dit dans un entretien avec Pierre Goldman (Libération, 8 mai 2014): «Fondamentalement je suis un journaliste. J'ai toujours été un journaliste. (...) Pour moi il n'y a pas de coupure entre le journalisme et la littérature, entre l'écriture romanesque et l'écriture journalistique ; même source, même objet, même problème : la réalité .» Mais Césaire n'est pas romancier...

5. Aimé Césaire, dans un débat de 1967 avec Jean-Marie Serreau, cité dans Veron et Hale, Les écrits d'Aimé Césaire, op.cit., p. 425.

6. James Arnold, Aimé Césaire, op.cit. p. 773.

7. Selon Alex Gil, «La représentation en profondeur de Et les chiens se taisaient d'Aimé Césaire ; pour une édition génétique en ligne », Genesis 33, "Afrique-Antilles ", p. 69. Il ne s'agit pas de modifications stylistiques mineures mais de déplacements par blocs de textes parfois considérables qui offrent une autre concaténation des faits.

8. "objective ", "scientifique " selon les critères des historiens occidentaux du XIX ème siècle souvent représentés par Fustel de Coulanges.

9. Et l'objet d'un essai de Césaire, Toussaint Louverture, la Révolution française et le problème colonial, Présence Africaine, 1960.

10. CRPN, p. 36.

11. Propos rapporté par Daniel Maximin dans Aimé Césaire, frère volcan, Éditions du Seuil, 2013, pp. 52-53

12. Frédéric Nietzsche, La naissance de la tragédie, 1872, Folio essais 1977, fragments posthumes 7 (23), p. 184.

13. James Arnold, Césaire, op.cit. p. 998.

14. Sur l'histoire de la mise en scène de TRC, voir James Arnold, Césaire, op.cit., pp. 987-999.

15. Interview à Fraternité-Matin du 29 avril 1966, cité dans Veron et Hale, Les écrits d'Aimé Césaire, op.cit., p. 393.

16. Interview par Claude Samuel, en ligne sur Théâtre total - Unesco, http:// unesdoc.unesco.org.

17. Le Rocado Zulu Théâtre est créé en 1977.

18. Dans une lettre à Guy Lenoir, metteur en scène, il lui demande de "l'espace sans décor » (Lettre du 13 sept. 1986).

19. M'appelle Mohamed Ali, Les solitaires intempestifs, 2014, p. 56. Dieudonné Niangouna, acteur et auteur, s'inscrit dans la filiation de Sony Labou Tansi.

\section{RÉSUMÉS}

Daniel Delas revient ici sur les abondantes réécritures que présente le théâtre de Césaire et explore cette hypothèse : c'est finalement la question du leader noir des indépendances afroantillaises et plus généralement celle de la libération de l'homme, qui hante Césaire pendant plus de trente ans et fait graviter l'ensemble de son œuvre, non seulement théâtrale, mais poétique et argumentative. Ce faisant, Césaire cherche à créer « un théâtre qui agit philosophiquement au niveau de l'homme tout entier ». 
Daniel Delas comes back to the profuse theatrical re-writings by Césaire and investigates the following hypothesis : it is finally the questionning about the black leader of Afro-Caribbean independencies and, more widely, about the human being emancipation that haunts Césaire during more than thirty years and remains the core of all his writings, non only theatrical ones, nut also poetic and argumentative. Thereby, Césaire tries to create "a theater acting philosophically at all levels of whole human being."

INDEX

Mots-clés : Césaire, théâtre de Césaire, génétique du théâtre de Césaire, Et les chiens se taisaient, indépendances afro-antillaises en littérature, La Tragédie du Roi Christophe

Keywords : Césaire, Césaire`s theater, Césaire`s theater genetics, Afro-Caribbean independencies in literaure, La Tragédie du Roi Christophe, Et les chiens se taisaient.

\section{AUTEUR}

DANIEL DELAS

Université de Cergy-Pontoise / ITEM 